PROCEEDINGS OF THE

AMERICAN MATHEMATICAL SOCIETY

Volume 130, Number 11, Pages 3301-3311

S 0002-9939(02)06480-8

Article electronically published on March 25, 2002

\title{
EXISTENCE THEORY FOR FIRST ORDER DISCONTINUOUS FUNCTIONAL DIFFERENTIAL EQUATIONS
}

\author{
EDUARDO LIZ AND RODRIGO L. POUSO \\ (Communicated by Carmen Chicone)
}

\begin{abstract}
We prove the existence of extremal solutions for a first order functional differential equation subject to nonlinear boundary conditions of functional type. Moreover, the functions that define our problem are allowed to be discontinuous. The proof of our main result is based on a generalized iterative technique.
\end{abstract}

\section{INTRODUCTION AND PRELIMINARIES}

We are concerned with the existence of solutions for first order scalar functional differential equations under weak regularity assumptions.

For fixed $r>0$ and $T>0$, the general form of a delay functional differential equation is

$$
x^{\prime}(t)=g\left(t, x_{t}\right) \quad \text { for all } t \in I=[0, T],
$$

where for every $t \in I$ and every $\xi \in \mathcal{C}([-r, T]), \xi_{t}$ denotes the element of $\mathcal{C}([-r, 0])$ defined by

$$
\xi_{t}(\theta):=\xi(t+\theta) \text { for all } \theta \in[-r, 0] .
$$

The function $\xi_{t}$ can be regarded as the history of $\xi$ from time $t-r$ up to time $t$.

In this setting, the function $g$ is usually assumed to be continuous on the set $I \times \mathcal{C}([-r, 0])$.

A first remarkable difference that one can find with respect to ordinary differential equations is that the Cauchy problem for equation (1.1) needs a functional initial condition, such as $x_{0}=\phi$, where $x_{0}$ is given by (1.2) with $t=0$ and $\phi \in \mathcal{C}([-r, 0])$ is the initial value. The reader is referred to [4, 5, 9] as outstanding monographs on functional differential equations.

On the other hand, and as it is observed in [2], the delay differential equation (1.1) can be studied in a more general frame of functional differential equations. Indeed, it suffices to define the function $f: I \times \mathcal{C}([-r, T]) \rightarrow \mathbb{R}$ as

$$
f(t, \xi):=g\left(t, \xi_{t}\right)
$$

Received by the editors July 10, 2000 and, in revised form, June 13, 2001.

1991 Mathematics Subject Classification. Primary 34A12, 34K07, 34K10.

Key words and phrases. Discontinuous functional differential equations, extremal solutions, existence results.

This research was partially supported by D.G.E.S. (Spain), projects PB97 - 0552 and HP19990026 .

(C)2002 American Mathematical Society 
in order to rewrite equation (1.1) as

$$
x^{\prime}(t)=f(t, x) \quad \text { for all } t \in I=[0, T] .
$$

Analogously, many other types of well-known functional differential equations can be reduced to an equation of the form of (1.3). Another example is furnished by the equations with maxima, which are of the type

$$
x^{\prime}(t)=h\left(t, x(t), \max _{s \in S(t)} x(s)\right) \quad \text { for all } t \in I=[0, T] .
$$

When $S(t)=[t-r, t]$, (1.4) can be regarded as a particular case of (1.1), but formulation (1.3) permits us to consider more general forms of $S(t) \subset \mathbb{R}$ (see, for instance [15, Eq. (1)]).

Equations with maxima have attracted much attention recently due to their importance in mathematical models such as the automatic control of technical systems (see e.g. [8, 14, 15, and references therein).

Monotone iterative techniques have been extensively applied to study the existence of solutions for delay differential equations and equations with maxima. See [11, 12 13, 17, where equation (1.1) is studied with periodic boundary conditions of "ordinary" type

$$
x(0)=x(T)
$$

and a constant initial condition $x(t)=x(0), t \in[-r, 0]$. The same problem is considered in [16 for (1.4) with $S(t)=[t-r, t]$. Moreover the right-hand side of the differential equation is assumed to be continuous in all of the above mentioned references.

For (1.4), Stepanov [15] considered a nonlinear two-point boundary condition of the form $h(x(0), x(T))=0$ and a Carathéodory function $f$.

We shall consider here a functional differential equation

$$
x^{\prime}(t)=f(t, x(t), x) \quad \text { for a.e. } t \in I=[0, T],
$$

with less restrictive conditions on $f$, in the spirit of 6 .

In relation to the boundary conditions, we would like to emphasize the fact that the usual pointwise boundary conditions which naturally arise in ordinary differential equations, do not seem to be so natural for functional differential equations. Indeed, if we regard the problem of finding a periodic solution as the problem of finding a fixed point for the Poincaré map, then the natural condition that appears in connection with equation (1.1) or (1.4) is

$$
x_{0}(\theta)=x_{T}(\theta) \text { for all } \theta \in[-r, 0] \text {, }
$$

instead of (1.5)). Condition (1.7) was considered in [3], where the monotone iterative method was developed for (1.1) with $f$ continuous.

We also remark that this periodic condition can be seen as a particular case of the more general functional boundary condition

$$
\mathcal{H}\left(x_{0}, x_{T}\right)=0
$$

for a given mapping $\mathcal{H}:(\mathcal{C}([-r, 0]))^{2} \rightarrow \mathcal{C}([-r, 0])$, proposed by Halanay [4]. The linear case of Halanay's boundary conditions $\mathcal{A}_{1} x_{0}+\mathcal{A}_{2} x_{T}=\Psi$, where $\mathcal{A}_{1}, \mathcal{A}_{2}$ : $\mathcal{C}([-r, 0]) \rightarrow \mathcal{C}([-r, 0])$ are given linear operators, was considered in [9, Chapter 11] and becomes (1.7) for $\mathcal{A}_{1}=-\mathcal{A}_{2}=I d, \Psi=0$. 
As far as the authors are aware, there is no previous work on existence of solutions for equation (1.1) or (1.4) together with condition (1.8) in the frame of upper and lower solutions. In this paper we consider the general functional boundary condition

$$
x(\theta)=B x(\theta) \text { for all } \theta \in[-r, 0],
$$

where $B$ is defined over a suitable set of functions $x:[-r, T] \rightarrow \mathbb{R}$ and need not be continuous. More precisely, for $r>0$ and $T>0$ fixed, we will define $B: \mathcal{S}_{r}^{T} \longrightarrow \mathcal{L}^{\infty}([-r, 0])$, where $\mathcal{L}^{\infty}([-r, 0])$ denotes the space of functions which are Lebesgue-measurable and bounded on $[-r, 0]$,

$$
\mathcal{S}_{r}^{T}=\left\{\xi:[-r, T] \rightarrow \mathbb{R}: \xi_{\mid[-r, 0]} \in \mathcal{L}^{\infty}([-r, 0]) \text { and } \xi_{\mid[0, T]} \in A C([0, T])\right\},
$$

and $A C([0, T])$ is the space of absolutely continuous functions on $[0, T]$. In $\mathcal{S}_{r}^{T}$ we consider the supremum norm

$$
\|\xi\|_{\infty}=\sup _{t \in[-r, T]}|\xi(t)| .
$$

Thus, we shall consider the following boundary value problem

$$
\left\{\begin{array}{l}
x^{\prime}(t)=f(t, x(t), x) \quad \text { for a.e. } t \in I=[0, T], \\
x(\theta)=B x(\theta) \text { for all } \theta \in[-r, 0],
\end{array}\right.
$$

subject to the following assumptions on the functions $f: I \times \mathbb{R} \times \mathcal{S}_{r}^{T} \longrightarrow \mathbb{R}$ and $B: \mathcal{S}_{r}^{T} \longrightarrow \mathcal{L}^{\infty}([-r, 0]):$

$(F)$ (f1) For all $(x, \xi) \in \mathbb{R} \times \mathcal{S}_{r}^{T}$ the function $f(\cdot, x, \xi)$ is measurable on $I$.

(f2) For a.e. $t \in I$ and all $(x, \xi) \in \mathbb{R} \times \mathcal{S}_{r}^{T}$ we have

$$
\limsup _{y \rightarrow x^{-}} f(t, y, \xi) \leq f(t, x, \xi) \leq \liminf _{y \rightarrow x^{+}} f(t, y, \xi) .
$$

(f3) For a.e. $t \in I$ and all $x \in \mathbb{R}$ the function $f(t, x, \cdot)$ is nondecreasing on $\mathcal{S}_{r}^{T}$.

(f4) For all $R>0$ there exists $\psi_{R} \in L^{1}(I)$ such that for a.e. $t \in I$ and for all $(x, \xi) \in \mathbb{R} \times \mathcal{S}_{r}^{T}$ with $|x| \leq R$ and $\|\xi\|_{\infty} \leq R$ we have

$$
|f(t, x, \xi)| \leq \psi_{R}(t) \text {. }
$$

(B) $B: \mathcal{S}_{r}^{T} \longrightarrow \mathcal{L}^{\infty}([-r, 0])$ is nondecreasing.

The aim of this paper is to prove a general existence result for problem $(P)$ under conditions $(F)$ and $(B)$ assuming the existence of lower and upper solutions. The proof of such a result is based upon an existence result for the Cauchy problem for ordinary differential equations and the generalized iterative techniques from [7].

An important point to note here is that this framework permits the study of some important cases of the above mentioned Halanay's boundary conditions as well as other different conditions. For instance, we can mention condition $U(x)=\gamma$, where $U: \mathcal{C}([0, T]) \rightarrow \mathbb{R}$ is a continuous linear form and $\gamma$ is a constant, suggested by Lee and O'Regan in [10]. They indicate the functional $U$ defined by $U(x)=\int_{0}^{T} x(s) d s$ as an example. It is obvious that in this case condition $U(x)=\gamma$ is a particular case of (1.9) with $B$ satisfying $(B)$.

Finally, several types of periodic conditions can be included in the formulation of problem $(P)$. For instance, the functional periodic condition (1.7) corresponds with $B \xi=\xi_{T}$ for $\xi \in \mathcal{S}_{r}^{T}$. The ordinary periodic conditions $x(0)=x(T)=x(t), t \in$ $[-r, 0]$ correspond with $B \xi(\theta)=\xi(T)$ for all $\theta \in[-r, 0]$. 
We divide our paper into three sections: in Section 2 we prove an existence result for the Cauchy problem for discontinuous ordinary differential equations, in Section 3 we establish and demonstrate our main existence result for the problem $(P)$, and, finally, in Section 4 we illustrate the applicability of our main result with two examples.

\section{INITIAL VALUE PROBLEMS}

Let $T>0$ be fixed and consider the initial value problem

$$
x^{\prime}(t)=f(t, x(t)) \text { for a.e. } t \in I=[0, T], x(0)=a .
$$

The following result is Theorem 3.1 in [6], and we include it here for the convenience of the reader.

Theorem 2.1. Assume that $f: I \times \mathbb{R} \longrightarrow \mathbb{R}$ satisfies the following conditions:

1. for all $x \in \mathbb{R}$ the function $f(\cdot, x)$ is measurable on $I$;

2. for a.e. $t \in I$ and all $x$ we have

$$
\limsup _{y \rightarrow x^{-}} f(t, y) \leq f(t, x) \leq \liminf _{y \rightarrow x^{+}} f(t, y)
$$

3. there exists $\psi \in L^{1}(I)$ such that for a.e. $t \in I$ and all $x \in \mathbb{R}$ we have

$$
|f(t, x)| \leq \psi(t)
$$

Then the initial value problem (2.1) has extremal solutions for every $a \in \mathbb{R}$.

Remark 2.2. Only the case $T=1$ and $a=0$ is considered in [6]. The reader can easily verify that the general case (i.e. $T \neq 1$ and/or $a \neq 0$ ) is reduced to that one by means of the change of variable $y(t)=x(t \cdot T)-a$ for all $t \in[0,1]$.

This last theorem can be extended by using lower and upper solutions.

Definition 2.3. We say that $\alpha$ is a lower solution of (2.1) if $\alpha \in A C(I)$,

$$
\alpha^{\prime}(t) \leq f(t, \alpha(t)) \quad \text { for a.e. } t \in I \quad \text { and } \alpha(0) \leq a \text {. }
$$

An upper solution is defined analogously reversing the inequalities.

Let $\alpha$ and $\beta$ be lower and upper solutions of 2.1) and $\alpha \leq \beta$. We say that a solution of (2.1), $x_{m}$, is the maximal solution of 2.1] in $[\alpha, \beta]$ if $x_{m} \in[\alpha, \beta]$ and $x_{m} \geq x$ for every solution $x$ of (2.1) in $[\alpha, \beta]$. In an analogous way we define the concept of minimal solution in $[\alpha, \beta]$ and when the minimal and maximal solutions in $[\alpha, \beta]$ exist, we call them extremal solutions in $[\alpha, \beta]$.

Theorem 2.4. Assume that $f: I \times \mathbb{R} \longrightarrow \mathbb{R}$ satisfies the following conditions:

1. for all $x \in \mathbb{R}$ the function $f(\cdot, x)$ is measurable on $I$;

2. for a.e. $t \in I$ and all $x$ we have

$$
\limsup _{y \rightarrow x^{-}} f(t, y) \leq f(t, x) \leq \liminf _{y \rightarrow x^{+}} f(t, y)
$$

3. for every $R>0$ there exists $\psi_{R} \in L^{1}(I)$ such that for a.e. $t \in I$ and all $x \in \mathbb{R}$ with $|x| \leq R$ we have

$$
|f(t, x)| \leq \psi_{R}(t)
$$

If $\alpha$ and $\beta$ are, respectively, a lower and an upper solution of problem (2.1) and $\alpha \leq \beta$, then the problem (2.1) has extremal solutions in $[\alpha, \beta]$. 
Proof. First, consider the modified problem

$$
x^{\prime}(t)=F(t, x(t)) \text { for a.e. } t \in I, x(0)=a,
$$

where

$$
F(t, x)=\left\{\begin{array}{cl}
\beta^{\prime}(t), & \text { if } x>\beta(t), \\
f(t, x), & \text { if } \alpha(t) \leq x \leq \beta(t), \\
\alpha^{\prime}(t), & \text { if } x<\alpha(t) .
\end{array}\right.
$$

Notice that every solution of (2.1) in $[\alpha, \beta]$ is a solution of (2.2).

The assumptions on $\alpha, \beta$ and $f$ ensure by Theorem 2.1 that problem (2.2) has extremal solutions.

Now we shall prove that every solution $x$ of $(2.2)$ belongs to $[\alpha, \beta]$ (which, in particular, implies that every solution of (2.2) is a solution of (2.1) in $[\alpha, \beta])$. We shall only demonstrate that $\alpha \leq x$, since the proof of $x \leq \beta$ is similar.

Assume, by contradiction, that there exist $t_{1}, t_{2} \in I$ such that $t_{1}<t_{2}$,

$$
\alpha\left(t_{1}\right)=x\left(t_{1}\right) \text { and } \alpha(t)>x(t) \text { for } t \in\left(t_{1}, t_{2}\right] .
$$

Since $x$ is a solution of (2.2) we have, by definition of $F$, that $x^{\prime}(t)=\alpha^{\prime}(t)$ for a.e. $t \in\left[t_{1}, t_{2}\right]$, and then

$$
\alpha\left(t_{2}\right)=\alpha\left(t_{1}\right)+\int_{t_{1}}^{t_{2}} \alpha^{\prime}(s) d s=x\left(t_{1}\right)+\int_{t_{1}}^{t_{2}} x^{\prime}(s) d s=x\left(t_{2}\right),
$$

which contradicts (2.3).

\section{MAIN RESULT}

In order to prove our main result, we need the following lemma, which is Theorem 1.2.2 in [7].

Lemma 3.1. Let $Y$ be a subset of an ordered metric space $X,[a, b]$ a nonempty interval in $Y$ and $G:[a, b] \rightarrow[a, b]$ a nondecreasing mapping.

If $\left\{G x_{n}\right\}$ converges in $Y$ whenever $\left\{x_{n}\right\} \subset[a, b]$ is a monotone sequence, then $G$ has a minimal fixed point $x_{*} \in[a, b]$ and a maximal one $x^{*} \in[a, b]$.

Moreover, these extremal fixed points satisfy the relations

$$
x_{*}=\min \{x \in[a, b]: G x \leq x\} \quad \text { and } \quad x^{*}=\max \{x \in[a, b]: G x \geq x\} .
$$

Now, to make a proper use of this lemma, we give some preliminaries about the space $\mathcal{S}_{r}^{T}$ defined in Section 1 . We can define a partial ordering on $\mathcal{S}_{r}^{T}$ as follows: for $\xi_{1}, \xi_{2} \in \mathcal{S}_{r}^{T}$ we write $\xi_{1} \leq \xi_{2}$ if and only if $\xi_{1}(t) \leq \xi_{2}(t)$ for all $t \in[-r, T]$. If $\xi_{1}, \xi_{2} \in \mathcal{S}_{r}^{T}$ and $\xi_{1} \leq \xi_{2}$, we define the functional interval

$$
\left[\xi_{1}, \xi_{2}\right]=\left\{\xi \in \mathcal{S}_{r}^{T}: \xi_{1} \leq \xi \leq \xi_{2}\right\} .
$$

It is easy to see that the intervals

$$
\left[\xi_{1}\right)=\left\{\xi \in \mathcal{S}_{r}^{T}: \xi_{1} \leq \xi\right\} \quad \text { and } \quad\left(\xi_{1}\right]=\left\{\xi \in \mathcal{S}_{r}^{T}: \xi_{1} \geq \xi\right\}
$$

are closed in $\mathcal{S}_{r}^{T}$, considering the supremum norm. Therefore $\left(\mathcal{S}_{r}^{T}, \leq,\|\cdot\|_{\infty}\right)$ is an ordered metric space (see [7]).

Next we introduce the concepts of lower and upper solution for problem $(P)$. 
Definition 3.2. We say that $\alpha:[-r, T] \rightarrow \mathbb{R}$ is a lower solution of $(P)$ if $\alpha \in \mathcal{S}_{r}^{T}$ and

$$
\left\{\begin{array}{l}
\alpha^{\prime}(t) \leq f(t, \alpha(t), \alpha) \text { for a.e. } t \in[0, T], \\
\alpha(\theta) \leq B \alpha(\theta) \text { for all } \theta \in[-r, 0] .
\end{array}\right.
$$

Analogously, we say that $\beta:[-r, T] \rightarrow \mathbb{R}$ is an upper solution of $(P)$ if $\beta \in \mathcal{S}_{r}^{T}$ and

$$
\left\{\begin{array}{l}
\beta^{\prime}(t) \geq f(t, \beta(t), \beta) \quad \text { for a.e. } t \in[0, T], \\
\beta(\theta) \geq B \beta(\theta) \text { for all } \theta \in[-r, 0] .
\end{array}\right.
$$

Finally, we say that $u$ is a solution of $(P)$ if it is both a lower and an upper solution.

The following theorem is our main result.

Theorem 3.3. Assume that conditions $(F)$ and $(B)$ are satisfied.

If there exist $\alpha$ and $\beta$, lower and upper solutions of $(P)$, and $\alpha \leq \beta$, then $(P)$ has extremal solutions in $[\alpha, \beta]$.

Proof. We shall only prove the existence of a minimal solution, since the arguments to show that there is a maximal one are analogous.

By condition (f4) there exists $\psi \in L^{1}(I)$ such that for a.e. $t \in I$, all $x \in$ $[\alpha(t), \beta(t)]$ and all $\xi \in[\alpha, \beta]$ we have

$$
|f(t, x, \xi)| \leq \psi(t) .
$$

Let us consider the mapping $G:[\alpha, \beta] \rightarrow[\alpha, \beta]$, where for a given $\xi \in[\alpha, \beta]$ the function $G \xi \in[\alpha, \beta]$ is defined as follows:

Definition of $G \xi$ on $[-r, 0]$. We define

$$
G \xi(\theta):=B \xi(\theta) \text { for all } \theta \in[-r, 0] .
$$

Notice that $\alpha \leq B \alpha \leq B \xi \leq B \beta \leq \beta$ on $[-r, 0]$ and thus $\alpha \leq G \xi \leq \beta$ on $[-r, 0]$.

Definition of $G \xi$ on $[0, T]$. By condition (f3), and since $\alpha(0) \leq B \xi(0) \leq \beta(0)$, the restrictions $\alpha_{\mid I}$ and $\beta_{\mid I}$ are, respectively, a lower and an upper solution (in the sense of Definition 2.3) of the initial value problem

$$
x^{\prime}(t)=f(t, x(t), \xi) \quad \text { for a.e. } t \in I, \quad x(0)=B \xi(0) .
$$

Hence we can apply Theorem 2.4 to ensure that (3.3) has extremal solutions between $\alpha_{\mid I}$ and $\beta_{\mid I}$.

We define $G \xi$ on $[0, T]$ as the minimal solution of (3.3) between $\alpha_{\mid I}$ and $\beta_{\mid I}$.

Since $G \xi_{\mid[-r, 0]} \in \mathcal{L}^{\infty}([-r, 0])$ and $G \xi_{\mid I} \in A C(I)$, then $G \xi \in \mathcal{S}_{r}^{T}$. Moreover, $\alpha \leq G \xi \leq \beta$ on $[-r, T]$ and, hence, $G \xi \in[\alpha, \beta]$.

Claim 1. $G$ is nondecreasing. Let $\xi_{1}, \xi_{2} \in[\alpha, \beta]$ be such that $\xi_{1} \leq \xi_{2}$. Since $B$ is nondecreasing, for all $\theta \in[-r, 0]$ we have that

$$
G \xi_{1}(\theta)=B \xi_{1}(\theta) \leq B \xi_{2}(\theta)=G \xi_{2}(\theta) .
$$

In particular, we have that $B \xi_{1}(0) \leq B \xi_{2}(0)$.

On the other hand, by the definition of $G$ and the condition (f3), for a.e. $t \in I$ we have that

$$
\left(G \xi_{2}\right)^{\prime}(t)=f\left(t, G \xi_{2}(t), \xi_{2}\right) \geq f\left(t, G \xi_{2}(t), \xi_{1}\right)
$$


which implies that $G \xi_{2 \mid I}$ is an upper solution (in the sense of Definition [2.3) of (3.3) with $\xi=\xi_{1}$. Moreover, $G \xi_{2} \geq \alpha$ on $I$ and $\alpha_{\mid I}$ is a lower solution of (3.3) with $\xi=\xi_{1}$. Thus, by Theorem 2.4 the problem (3.3) with $\xi=\xi_{1}$ has solutions between $\alpha_{\mid I}$ and $G \xi_{2 \mid I}$. Finally, since $G \xi_{1 \mid I}$ is the minimal solution of that problem between $\alpha_{\mid I}$ and $\beta_{\mid I}$, we conclude that $G \xi_{1} \leq G \xi_{2}$ on $I$.

Claim 2. $\left\{G \xi_{n}\right\}$ converges in $\mathcal{S}_{r}^{T}$ whenever $\left\{\xi_{n}\right\} \subset[\alpha, \beta]$ is a monotone sequence. Let $\left\{\xi_{n}\right\} \subset[\alpha, \beta]$ be a monotone sequence. Since $G$ is nondecreasing and maps $[\alpha, \beta]$ into itself, then $\left\{G \xi_{n}\right\}$ is monotone and bounded. Hence we can define

$$
y(t)=\lim _{n \rightarrow \infty} G \xi_{n}(t) \quad \text { for all } t \in[-r, T] .
$$

Obviously, $\alpha \leq y \leq \beta$ on $[-r, T]$. Let us prove that $y \in \mathcal{S}_{r}^{T}$.

First, $y_{\mid[-r, 0]}$ is measurable on $[-r, 0]$ because it is a pointwise limit of measurable functions on $[-r, 0]$. Thus $y_{\mid[-r, 0]} \in \mathcal{L}^{\infty}([-r, 0])$.

On the other hand, by (3.1) and the definition of $G \xi_{n}$, for all $n \in \mathbb{N}$ and all $t, s \in[0, T]$ we have

$$
\left|G \xi_{n}(t)-G \xi_{n}(s)\right| \leq\left|\int_{s}^{t} \psi(r) d r\right|
$$

which implies that for all $t, s \in[0, T]$ we have

$$
|y(t)-y(s)| \leq\left|\int_{s}^{t} \psi(r) d r\right| .
$$

Therefore $y_{\mid I} \in A C(I)$ and, thus, $y \in \mathcal{S}_{r}^{T}$.

By Lemma 3.1 $G$ has a minimal fixed point $x_{*} \in[\alpha, \beta] \cap Y$ which satisfies

$$
x_{*}=\min \{\xi \in[\alpha, \beta] \cap Y: G \xi \leq \xi\} .
$$

It is easy to verify that the fixed points of $G$, and in particular $x_{*}$, are solutions of $(P)$.

Claim 3. $x_{*}$ is the minimal solution of $(P)$ in $[\alpha, \beta]$. Assume that $v \in[\alpha, \beta]$ is a solution of $(P)$. Since $v$ is a solution of $(P)$, we have that $v(\theta)=B v(\theta)=G v(\theta)$ for all $\theta \in[-r, 0]$. Moreover, $v$ is a solution between $\alpha_{\mid I}$ and $\beta_{\mid I}$ of the initial value problem (3.3) with $\xi=v$, hence $v(t) \geq G v(t)$ for all $t \in I$, because $G v_{\mid I}$ is the minimal solution of that problem between $\alpha_{\mid I}$ and $\beta_{\mid I}$. Therefore we conclude that $G v \leq v$ on $[-r, T]$ which, together with (3.4), implies that $x_{*} \leq v$ on $[-r, T]$.

Remark 3.4. Notice that the above proof holds if we replace $\mathcal{S}_{r}^{T}$ by the larger set

$$
\begin{aligned}
\tilde{\mathcal{S}_{r}^{T}}=\{\xi:[-r, T] \rightarrow & \mathbb{R}: \xi_{\mid[-r, 0]} \text { bounded on }[-r, 0] \text { and } \\
& \left.\xi_{\mid[0, T]} \in A C([0, T])\right\} .
\end{aligned}
$$

We have preferred to establish our result with $\mathcal{S}_{r}^{T}$ because some interesting boundary operators $B$ may fail to be defined over $\tilde{\mathcal{S}_{r}^{T}}$. For instance, the operator $B$ : $\mathcal{S}_{r}^{T} \rightarrow \mathcal{L}^{\infty}([-r, 0])$ given by

$$
B \xi(\theta)=\int_{-r}^{T / 2} \xi(s) d s \quad \text { for all } \theta \in[-r, 0] \text { and all } \xi \in \mathcal{S}_{r}^{T},
$$

cannot be extended to $\tilde{\mathcal{S}_{r}^{T}}$. 


\section{EXAMPLES}

In this section, we illustrate our main result with two examples. The first one shows that we can prove the existence of extremal solutions for problems not covered by previous known results. The second one is a motivation from the real life for our study.

Example 4.1. Consider the periodic problem

$$
\begin{gathered}
x^{\prime}(t)=\frac{\operatorname{Tanh}\left(\left[\max _{s \in[t-r, t]} x(s)\right]\right)-x(t)}{\sqrt{t}}+[x(t)]+\operatorname{sgn}(x(t)) \text { for a.e. } t \in I, \\
x_{0}=x_{1},
\end{gathered}
$$

where $I=[0,1], r \in(0,1)$ is fixed and

$$
\xi_{t}(\theta):=\xi(t+\theta) \quad \text { for all } \theta \in[-r, 0] \text { and all } \xi \in \mathcal{S}_{r}^{1} .
$$

In the equation, "Tanh" stands for the hyperbolic tangent, square brackets mean integer part, and $\operatorname{sgn}(x)=x /|x|$ for $x \neq 0, \operatorname{sgn}(0)=0$.

Problem (4.1) - (4.2) falls inside the scope of Theorem 3.3 To make this fact clearer, it suffices to define $f:[0,1] \times \mathbb{R} \times \mathcal{S}_{r}^{1} \rightarrow \mathbb{R}$ and $B: \mathcal{S}_{r}^{1} \rightarrow \mathcal{L}^{\infty}([-r, 0])$ in such a way that

$$
\begin{gathered}
f(t, x, \xi):=\frac{\operatorname{Tanh}\left(\left[\sup _{s \in[t-r, t]} \xi(s)\right]\right)-x}{\sqrt{t}}+[x]+\operatorname{sgn}(x), \quad \text { for } t \neq 0, \\
B \xi(\theta):=\xi(1+\theta) \quad \text { for all } \theta \in[-r, 0] \text { and all } \xi \in \mathcal{S}_{r}^{1} .
\end{gathered}
$$

Note that $f$ is discontinuous with respect to all of its variables. In particular, it is discontinuous with respect to $x$ and $\xi$ and therefore none of the existence results given in [3, 10, 11, 12, 13, 15, 16, 17] is suitable for this problem. However, $f$ fulfills conditions $(F)$.

We also note that some existence results on this type of equations have been recently proven. In [1] the authors also consider functional-boundary conditions. However, the functional periodic conditions that we consider in this example are not covered by the main result in [1] because the assumptions that are required on the corresponding function $B$ are rather strong. In particular, the reader can check that condition (B1) in 1] does not hold when trying to apply Theorem 3.1 in [1] to (4.1)-(4.2) .

One can readily verify that for all $t \in(0,1]$, for all $x \in \mathbb{R}$ and for all $\xi \in \mathcal{S}_{r}^{1}$ we have

$$
p(t) x+N_{1}(t) \leq f(t, x, \xi) \leq p(t) x+N_{2}(t),
$$

for

$$
p(t)=1-\frac{1}{\sqrt{t}}, \quad N_{1}(t)=-2+\frac{1}{t}, \quad N_{2}(t)=1+\frac{1}{\sqrt{t}} .
$$


Now let $\alpha:[-r, 1] \rightarrow \mathbb{R}$ be such that $\alpha_{\mid[0,1]}$ is the unique solution of the linear scalar periodic problem

$$
\left\{\begin{array}{c}
\alpha^{\prime}(t)=p(t) \alpha(t)+N_{1}(t) \quad \text { for a.e. } t \in I \\
\alpha(0)=\alpha(1)
\end{array}\right.
$$

and let $\alpha$ be extended to $[-r, 1]$ in such a way that $\alpha_{0}=\alpha_{1}$. It is important to note that the explicit expression for $\alpha$ can be easily obtained. We define $\beta:[-r, 1] \rightarrow \mathbb{R}$ in an analogous way but we replace $N_{1}$ by $N_{2}$.

Now, the inequalities (4.3) imply that $\alpha$ and $\beta$ are respectively a lower and an upper solution for problem (4.1)- (4.2). Furthermore, it can be proven that $\alpha \leq \beta$ on $[0,1]$ just by using the fact that

$$
\int_{0}^{1} p(s) d s<0
$$

as the reader can see in [7, Lemma 1.5.6]. Hence $\alpha \leq \beta$ on $[-r, 1]$.

Example 4.2. Our second example is a model for compound interest. Suppose that a sum of money $S_{0}>0$ is deposited in a bank that pays an interest $\mathcal{I} \in(0,1)$. A well-known and simple model for describing the evolution of the money with time is

$$
S^{\prime}(t)=\mathcal{I} S(t),
$$

where $S(t)$ stands for the sum of money at time $t>0$.

The key assumptions that lead to this model are that interest does not change with time and that it is compounded continuously.

However, it is quite reasonable to think that interest is likely to change with time and even with the amount of money deposited in the account. Let us consider the following assumptions:

1. The bank assigns to each account an interest which increases with the amount of money that it contains. Suppose that the bank acts as follows: the first day of every month the interest is revised in terms of the mean value of the money in the account during the preceding month (banks prefer steady customers, that is why it seems more appropriate to take into account a mean value rather than a punctual datum). Suppose also that no revision of this type is made until the first day of the next month.

If time $t$ is computed in months we may assume, for simplicity, that nonnegative integers $t=0,1,2, \ldots$, correspond with the instants when revisions are made (in particular, we are considering that all months have the same number of days, the reader who feels uneasy with this convention may replace months by, say, fortnights). Therefore, at a month $t>0$ the interest that is being paid by the bank is a function of

$$
\text { mean value of } S(t) \text { in the preceding month }=\int_{[t]-1}^{[t]} S(r) d r
$$

where $[t]$ stands for the integer part of $t$.

We note that the bank can only provide a finite number of different types of interest. Therefore the interest is necessarily a discontinuous function. 
2. On the other hand, interest may change with time (due to changes in economy, sudden variations in the bank's policy, and so on). As we all know, this type of change may be rather abrupt, which indicates that continuous dependence with time is a rather unrealistic assumption.

Assumptions 1 and 2 lead to the following model:

$$
\begin{array}{r}
S^{\prime}(t)=\mathcal{I}\left(t, \int_{[t]-1}^{[t]} S(r) d r\right) S(t), \quad t>0, \\
S(\theta)=S_{0}(\theta), \quad \theta \in[-1,0],
\end{array}
$$

where $S_{0} \in \mathcal{L}^{\infty}([-1,0])$ is the (known) evolution of money during the first month and $\mathcal{I}:[0, \infty) \times \mathbb{R} \rightarrow(0,1)$ satisfies the following conditions:

(i) For each $m \in \mathbb{R}, \mathcal{I}(\cdot, m)$ is measurable on $[0, \infty)$;

(ii) For a.e. $t \in[0, \infty), \mathcal{I}(t, \cdot)$ is nondecreasing on $\mathbb{R}$.

We emphasize the lack of continuity of $\mathcal{I}$ with respect to both of its variables, which makes it impossible to study problem (4.4)-4.5) from the point of view of some recent books on functional differential equations as [5, 9]. It is also necessary to remark that continuity is precisely a bad assumption in the situation we are trying to study, as we already mentioned in 1 and 2 .

In order to study the existence of solutions for the problem 4.4 -4.5 by using Theorem 3.3, we fix a constant $T>0$ and we define

$$
f:[0, T] \times \mathbb{R} \times \mathcal{S}_{1}^{T} \longrightarrow \mathbb{R}
$$

as

$$
f(t, x, \xi)=\mathcal{I}\left(t, \int_{[t]-1}^{[t]} \xi(s) d s\right) x .
$$

Note that the mapping

$$
t \in[0, T] \mapsto \int_{[t]-1}^{[t]} \xi(s) d s,
$$

is piecewise constant for every fixed $\xi \in \mathcal{S}_{1}^{T}$, hence we can express

$$
\mathcal{I}\left(t, \int_{[t]-1}^{[t]} \xi(s) d s\right),
$$

as a finite sum of measurable functions and therefore the mapping

$$
t \in[0, T] \mapsto f(t, x, \xi)
$$

is measurable on $[0, T]$ for fixed $x \in \mathbb{R}$ and $\xi \in \mathcal{S}_{1}^{T}$. It is easy to verify that $f$ satisfies the remaining conditions.

On the other hand, we define $B: \mathcal{S}_{1}^{T} \rightarrow \mathcal{L}^{\infty}([-1,0])$ as the constant mapping $B \xi=S_{0}$.

Finally, since $\mathcal{I} \in(0,1)$, it is easy to check that

$$
\beta(t)= \begin{cases}S_{0}(t), & t \in[-1,0], \\ S_{0}(0) \mathrm{e}^{t}, & t \in[0, T],\end{cases}
$$


is an upper solution for (4.4)- (4.5) and that

$$
\alpha(t)= \begin{cases}S_{0}(t), & t \in[-1,0], \\ S_{0}(0), & t \in[0, T],\end{cases}
$$

is a lower one.

Theorem 3.3 ensures that (4.4)-4.5) has extremal solutions between $\alpha$ and $\beta$.

\section{REFERENCES}

[1] S. Carl and S. Heikkilä, Extremality results for first-order discontinuous functional differential equations, Comput. Math. Appl. 40 (2000), 1217-1232. MR 2001g:34095

[2] A. Fryszkowski, Existence of solutions of functional-differential inclusions in nonconvex case, Ann. Polon. Math. 45 (1985), 121-124. MR 86i:34018

[3] J. R. Haddock and M. N. Nkashama, Periodic boundary value problems and monotone iterative methods for functional differential equations, Nonlinear Anal. 22, 3 (1994), 267-276. MR 95h:34102

[4] A. Halanay, "Differential equations: Stability, oscillations, time lags", Academic Press, New York (1966). MR 35:6938

[5] J. K. Hale and S. M. Verduyn Lunel "Introduction to functional-differential equations", Springer-Verlag, New York (1993). MR 94m:34169

[6] E. R. Hassan and W. Rzymowski, Extremal solutions of a discontinuous differential equation, Nonlinear Anal. 37 (1999), 997-1017. MR 2000d:34015

[7] S. Heikkilä and V. Lakshmikantham, "Monotone iterative techniques for discontinuous nonlinear differential equations", Marcel Dekker, New York (1994). MR 95d:34002

[8] A. Ivanov, E. Liz and S. Trofimchuk, Halanay inequality, Yorke 3/2 stability criterion, and differential equations with maxima, Tohoku Math.J., to appear.

[9] V. Kolmanovskii and A. Myshkis, "Introduction to the theory and applications of functionaldifferential equations", Kluwer Academic Publishers, Dordrecht (1999). MR 2000c:34164

[10] J. W. Lee and D. O'Regan, Existence results for differential delay equations, I. J. Differential Equations 102 (1993), 342-359. MR 94c:34096

[11] S. Leela and M.N. Oguztöreli, Periodic boundary value problem for differential equations with delay and monotone iterative method. J. Math. Anal. Appl. 122 (1987), 301-307. MR 88d:34095

[12] X. Liu, Periodic boundary value problems for differential equations with finite delay. Dynam. Systems Appl. 3 (1994), 357-367. MR 95f:34096

[13] E. Liz and J. J. Nieto, Periodic boundary value problems for a class of functional-differential equations, J. Math. Anal. Appl. 200 (1996), 680-686. MR 97k:34097

[14] M. Pinto and S. Trofimchuk, Stability and existence of multiple periodic solutions for a quasilinear differential equation with maxima. Proc. Roy. Soc. Edimburgh Sect. A 130 (2000), 1103-1118. MR 2001j:34101

[15] E. Stepanov, On solvability of some boundary value problems for differential equations with "maxima", Topol. Methods Nonlinear Anal. 8 (1996), 315-326. MR 99a:34178

[16] H. K. Xu and E. Liz, Boundary value problems for differential equations with maxima, Nonlinear Stud. 3, 2 (1996), 231-241.

[17] H. K. Xu and E. Liz, Boundary value problems for functional differential equations, Nonlinear Anal. 41 (2000), 971-988. MR 2001d:34111

Departamento de Matemática Aplicada, E.T.S.E. Telecomunicación, Universidade de Vigo, Campus Marcosende, 36280 Vigo, Spain

E-mail address: eliz@dma.uvigo.es

Departamento de Análise Matemática, Facultade de Matemáticas, Campus Sur, Universidade de Santiago de Compostela, 15782 Santiago de Compostela, Spain

E-mail address: rodrigolp@correo.usc.es 